Vietnam Journal of Mechanics, VAST, Vol.31, No. 3 \&4 (2009), pp. $191-210$

\title{
CYCLIC BEHAVIOR AND ENERGY APPROACH OF THE FATIGUE OF SHAPE MEMORY ALLOYS
}

\author{
${ }^{1}$ Ziad Moumni, ${ }^{2}$ Wael Zaki, ${ }^{3}$ Habibou Maitournam \\ ${ }^{1}$ UME-MS, École Nationale Supérieure de Techniques Avancées \\ 91761 Palaiseau Cedex, France \\ ${ }^{2}$ LMS, École Polytechnique, 91128 Palaiseau Cedex, France
}

\begin{abstract}
This paper presents an energy-based low-cycle fatigue criterion that can be used in analyzing and designing structures made from shape memory alloys (SMAs) subjected to cyclic loading. Experimentally, a response similar to plastic shakedown is observed: during the first cycles the stress-strain curve shows a hysteresis loop which evolves during the first few cycles before stabilizing. By adopting an analogy with plastic fatigue, it is shown that the dissipated energy of the stabilized cycle is a relevant parameter for estimating the number of cycles to failure of such materials. Following these observations, we provide an application of the cyclic model, previously developed by the authors within the framework of generalized standard materials with internal constraints [16], in order to evaluate such parameter. Numerical simulations are presented along with a validation against experimental data in case of cyclic superelasticity.

Keywords: cyclic loading, residual strain, internal stress, dissipation, fatigue.
\end{abstract}

\section{INTRODUCTION}

The interesting behavior of SMAs is essentially due to their capability of undergoing a reversible diffusionless solid-solid phase transition known as "the martensitic transformation" $[31,24,18]$. This transition is characterized at the microscopic level by a modification of the crystallographic lattice structure, which can be induced by altering either the material temperature or the stress to which it's subjected or both, hence a strong thermomechanical coupling. At high temperature, a shape memory alloy consists of a relatively ordered parent phase called austenite, which transforms when cooled into a less ordered product phase called martensite. In the absence of stress, this leads to "selfaccommodation" of martensite plates, i.e. to the formation of lattice twins without any macroscopic deformation.

Mechanical loading may lead to detwinning of self-accommodating martensite. In this case, martensite plates become oriented according to privileged directions that depend on the applied stress. The resulting inelastic macroscopic strain usually reaches several percent; it can be recovered by heating, in which case the SMA regains its initial undeformed austenitic shape. Simple way shape memory refers to the ability of a shape memory alloy to remember its high temperature state. 
Beside the characteristic shape memory behavior SMAs exhibit other interesting effects, namely: superelasticity or pseudoelasticity, which is the ability of a shape memory alloy to accommodate large strains due to stress-induced phase change at a constant, sufficiently high temperature and to recover its undeformed shape upon unloading; and the superthermal effect, which is the ability to deform an initially austenitic SMA by cooling under constant stress and then to recover the austenitic shape by heating. The magnitude of the temperature-induced strains depend on the applied stress.

Furthermore, cyclic loading may allow SMAs to have a "two-way shape memory effect". In this case, the material can change its shape reversibly due to cyclic heatingcooling.

Since components made of SMAs usually operate under cyclic thermomechanical loading; their design requires reliable prediction of the material's cyclic 3D response and fatigue resistance. In this regard, several models exist that are capable of handling cyclic, mainly superelastic, SMA behavior [9, 1, 8, 26, 7, 35, 34, etc.].

The interesting properties of SMAs promoted their use in several fields, especially in outer space (antennas, braces) and in medical applications (orthodontia, cardiology, implants miniaturization, etc.). SMAs are also becoming increasingly attractive for automotive, nuclear and civil engineering applications, mainly due to their high damping capacity.

One of the main difficulties facing their use in technologically advanced applications with high security specifications concern the poorly known fatigue behavior of these alloys as well as the amnesia phenomena encountered with shape memory properties. A better knowledge and control of these two aspects should promote their use. Two types of fatigue have to be considered :

First, classical mechanical fatigue due to mechanical cycling in the pseudoelastic domain $[14,29]$. The objective is to determine the number of cycles before failure. For instance, SMA are used in the biomedical field to manufacture stents, endovascular prosthesis inserted in blood vessels to avoid thrombosis and occlusion of the vessels. In stents, cyclic loads would arise from the difference in systolic and diastolic blood pressures and from the stress associated with the contraction of the heart muscle (e.g. see [10]). It is of primary importance to know the number of cycles before any damage occur to the stent.

Second, thermal fatigue or amnesia of the material, due to a degradation of the material characteristics responsible for the shape memory effect, like the transformation temperatures. The question is to determine if the material remains able to remember its initial shape.

Fatigue of shape memory alloys has also attracted considerable attention $[11,12,13$, $30,28,21]$; it is still, however, not very well understood. Particularly, fatigue mechanisms at the microscopic level are still being investigated [25, 22]. Nevertheless, Manson-Coffingtype criteria have been successfully used for predicting fatigue induced failure of simple SMA structures subjected to uniaxial loading $[11,25,30]$. Like in classical elastoplastic materials, such as steel, the number of cycles to failure of a SMA varies depending on its composition and on the applied loading, among other factors. This number may range from $10^{4}$ cycles for thermal valves using one-way shape memory effect [4] to a nominal $4 \times 10^{8}$ cycles for stents [15]. 
Using the analogy with plastic fatigue (low-cycle fatigue) [5, 3], [17] established a relation between the amount of dissipated energy associated with the stabilized hysteresis cycle and the number of cycles to failure. In this paper, we provide an application of the cyclic model, developed previously by the authors within the framework of generalized standards materials with internal constraints [18], in order to simulate the dissipated energy at the stabilized cycle. Our aim here is to shown that the cyclic model can be combined with the fatigue criterion in order to predict low-cycle failure of superelastic shape memory structures.

The paper is organized as follows:

First, section (2) is devoted to the presentation of the cyclic Zaki-Moumni model where the behavior is described using three state variables representing residual strain induced by cyclic loading, internal stress induced by repeated phase change and the cumulated martensite volume fraction. The reader is referred to [33, 34] for a detailed discussion of the model.

Second, the low cycle fatigue law and numerical simulations of the dissipated energy at the stabilized cycle along with a validation against experimental data in case of cyclic superelasticity are presented in section (3).

The conclusion of the work is given in section (4).

\section{THE CYCLIC MODEL OF SMA BEHAVIOR}

\subsection{Experimental observations}

Figure 1 illustrates the response of a Nickel-Titanium wire to repeated tension. Some observations can be made:

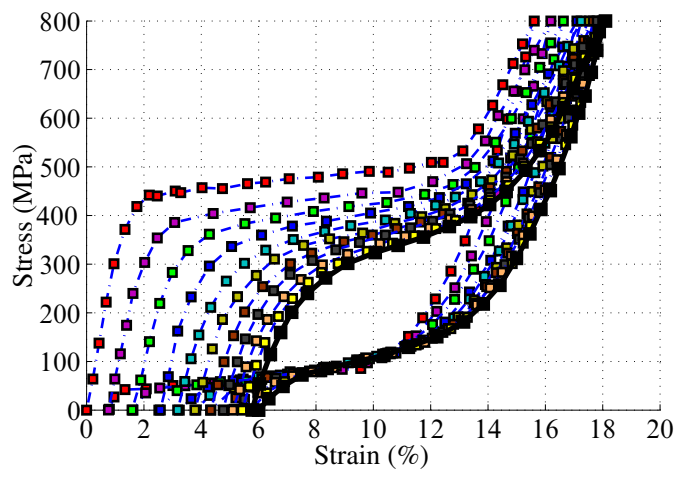

Fig. 1. Cyclic superelastic tensile response of a NiTi wire. The characteristic hysteresis loop tends to stabilize when the number of cycles increases

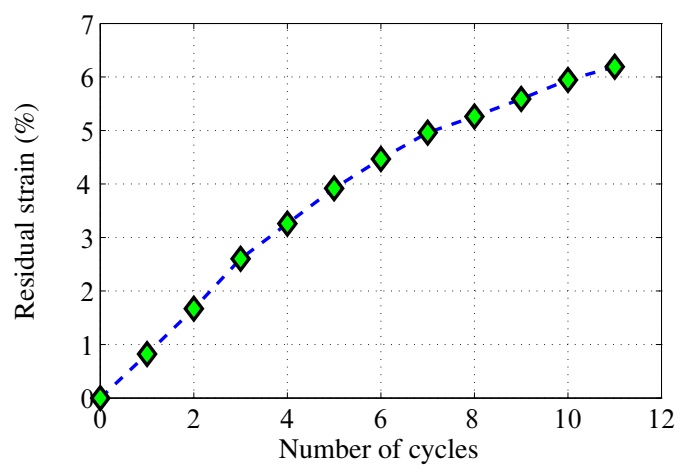

Fig. 2. Residual strain vs the number of cy

- Recovery of inelastic strain is not complete at the end of each cycle. Indeed, after complete unloading, some residual strain remains. This strain increases exponentially with the number of cycles, as shown in figure 2 . 
- Forward phase change yield stress decreases with increasing number of cycles (figure 3).

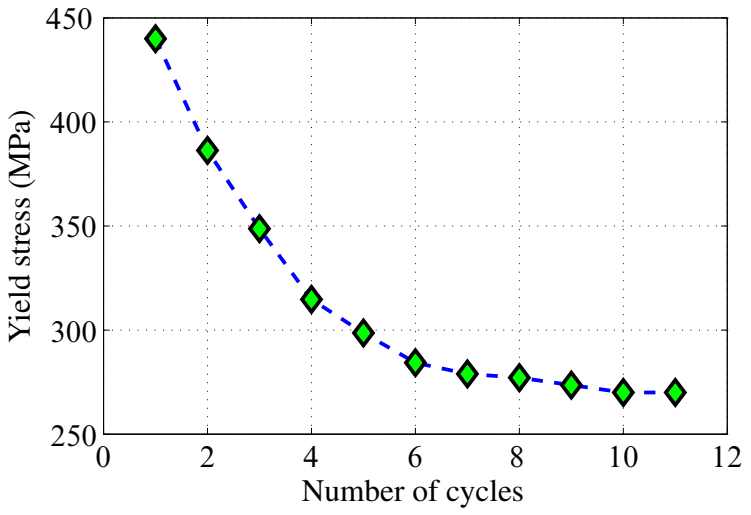

Fig. 3. Forward phase change yield stress vs the number of tensile loading cycles

- The hysteresis loop evolves progressively with the number of cycles before stabilizing. In figure 1, the stabilized loop is shown in continuous dark line.

Residual strain is generally considered to be due to some oriented martensite not transforming back into austenite during reverse phase change [7, 2]. Repeated forward and reverse phase changes create some defects within the material [1], which result in localized internal stresses [26], allowing SMAs to exhibit two-way shape memory, the material is said to be trained (training phenomena). The internal stress eliminates the need for external loading in order to orient martensite variants. As a result, the shape memory structure can assume two different shapes when temperature varies: an austenitic undeformed shape at high temperature and a deformed low-temperature shape resulting from martensite orientation due to internal stress.

\subsection{Phenomenological model}

For full details regarding the model presented in this section, the reader is referred to $[32,33,34][18]$.

As seen in the previous section, macroscopic cyclic response of superelastic SMAs induces residual inelastic strains and localized internal stresses within the material. Hence, two state variables are introduced: a residual strain tensor $\varepsilon_{\mathrm{r}}$ and an internal stress tensor $\boldsymbol{B}$. A third variable, $z_{\mathrm{e}}$, representing cumulated martensite volume fraction is also used:

$$
z_{\mathrm{e}}=\int_{0}^{t}|\dot{z}| d \tau
$$

where $t$ is a kinematic time. The effect of cyclic loading on the material parameters can be modeled by considering these parameters to depend on $z_{\mathrm{e}}$.

\subsubsection{State variables and free energy}

The following state variables are considered:

- Macroscopic strain $\varepsilon$ and temperature $T$; 
- Volume fraction $z$ and cumulated volume fraction $z_{\mathrm{e}}$ of martensite;

- Local strain tensors: $\varepsilon_{\mathrm{A}}$ for austenite and $\varepsilon_{\mathrm{M}}$ for martensite;

- Local martensite transformation strain tensor $\varepsilon_{\text {tr }}$;

- Internal stress $\boldsymbol{B}$ and residual strain $\varepsilon_{\mathrm{r}}$.

Phase change latent heat is assumed to depend on the cumulated fraction $z_{\mathrm{e}}$. Respective free energy densities of austenite and martensite are taken to be

$$
\mathcal{W}_{\mathrm{A}} \stackrel{\text { def }}{=} W_{\mathrm{A}}\left(\varepsilon_{\mathrm{A}}, \varepsilon_{\mathrm{r}}\right)=\frac{1}{2}\left(\varepsilon_{\mathrm{A}}-\varepsilon_{\mathrm{r}}\right): \boldsymbol{K}_{\mathrm{A}}:\left(\varepsilon_{\mathrm{A}}-\varepsilon_{\mathrm{r}}\right)
$$

and

$$
\begin{aligned}
\mathcal{W}_{\mathrm{M}} & =W_{\mathrm{M}}\left(\varepsilon_{\mathrm{M}}, \varepsilon_{\mathrm{tr}}, \varepsilon_{\mathrm{r}}, T, \boldsymbol{B}, z_{\mathrm{e}}\right) \\
& =\frac{1}{2}\left(\varepsilon_{\mathrm{M}}-\varepsilon_{\mathrm{tr}}-\varepsilon_{\mathrm{r}}\right): \boldsymbol{K}_{\mathrm{M}}:\left(\varepsilon_{\mathrm{M}}-\varepsilon_{\mathrm{tr}}-\varepsilon_{\mathrm{r}}\right)+C\left(T, z_{\mathrm{e}}\right)-\frac{2}{3} \boldsymbol{B}: \varepsilon_{\mathrm{tr}}
\end{aligned}
$$

$\boldsymbol{B}: \varepsilon_{\mathrm{tr}}$ in the expression of $\mathcal{W}_{\mathrm{M}}$ allows modeling the two-way shape memory effect. It represents a modification of the free energy of martensite due to the creation of internal stresses, which allows austenite to transform more easily into oriented martensite. Subsequent sections will help clarify this idea, especially when phase change criteria are established.

The contribution of austenite-martensite interaction to the SMA free energy density is assumed to be

$$
\mathcal{I}=I\left(z, z_{\mathrm{e}}, \varepsilon_{\mathrm{tr}}\right)=G \frac{z^{2}}{2}+\frac{z}{2}[\alpha z+\beta(1-z)]\left(\frac{2}{3} \varepsilon_{\mathrm{tr}}: \varepsilon_{\mathrm{tr}}\right)
$$

$G, \alpha$ and $\beta$ are material parameters functions of the cumulated martensite volume fraction $z_{\mathrm{e}}\left(G=\hat{G}\left(z_{\mathrm{e}}\right), \quad \alpha=\hat{\alpha}\left(z_{\mathrm{e}}\right)\right.$ and $\left.\beta=\hat{\beta}\left(z_{\mathrm{e}}\right)\right)$ :

- $\beta \frac{z(1-z)}{2}\left(\frac{2}{3} \varepsilon_{\mathrm{tr}}: \varepsilon_{\mathrm{tr}}\right)$ represents interaction between austenite and martensite.

Following many published works $[6,23,19$, etc.], this interaction is taken to be proportional to the volume fractions of interacting phases. $\beta$ determines how a mechanical loading applied to an initially austenitic shape memory material affects martensite orientation during phase change;

- $G \frac{z^{2}}{2}$ quantifies orientation-independent interaction between martensite variants;

- Finaly, $\alpha \frac{z^{2}}{2}\left(\frac{2}{3} \varepsilon_{\mathrm{tr}}: \varepsilon_{\mathrm{tr}}\right)$ accounts for interaction increase due to orientation of martensite plates; its expression is similar to that of the energy contribution due to linear kinematic hardening of an elastoplastic material with hardening coefficient $\alpha . \alpha$ controls the slope of the stress-strain curve corresponding to martensite orientation. 
Finally, the free energy density of the material is given by

$$
\begin{aligned}
\mathcal{W} \stackrel{\text { def }}{=} W\left(\varepsilon, T, \varepsilon_{\mathrm{A}}, \varepsilon_{\mathrm{M}}, z, \varepsilon_{\mathrm{tr}}, \varepsilon_{\mathrm{r}}, \boldsymbol{B}, z_{\mathrm{e}}\right) \\
=(1-z)\left[\frac{1}{2}\left(\varepsilon_{\mathrm{A}}-\varepsilon_{\mathrm{r}}\right): \boldsymbol{K}_{\mathrm{A}}:\left(\varepsilon_{\mathrm{A}}-\varepsilon_{\mathrm{r}}\right)\right] \\
+z\left[\frac{1}{2}\left(\varepsilon_{\mathrm{M}}-\varepsilon_{\mathrm{tr}}-\varepsilon_{\mathrm{r}}\right): \boldsymbol{K}_{\mathrm{M}}:\left(\varepsilon_{\mathrm{M}}-\varepsilon_{\mathrm{tr}}-\varepsilon_{\mathrm{r}}\right)+C\left(T, z_{\mathrm{e}}\right)\right] \\
+G \frac{z^{2}}{2}+\frac{z}{2}[\alpha z+\beta(1-z)]\left(\frac{2}{3} \varepsilon_{\mathrm{tr}}: \varepsilon_{\mathrm{tr}}\right)-\frac{2}{3} z \boldsymbol{B}: \varepsilon_{\mathrm{tr}} .
\end{aligned}
$$

\subsubsection{Internal constraints and Lagrangian}

State variables obey the following constraints:

- Martensite volume fraction is necessarily within the $[0,1]$ interval.

$$
\begin{array}{r}
z \geqslant 0, \\
(1-z) \geqslant 0
\end{array}
$$

- The equivalent transformation strain $\sqrt{\frac{2}{3} \varepsilon_{\mathrm{tr}}: \varepsilon_{\mathrm{tr}}}$ has a maximum value $\gamma$ that varies with respect to the cumulated volume fraction $z_{\mathrm{e}}$.

$$
\gamma-\sqrt{\frac{2}{3} \varepsilon_{\mathrm{tr}}: \varepsilon_{\mathrm{tr}}} \geqslant 0, \quad \gamma \stackrel{\text { def }}{=} \hat{\gamma}\left(z_{\mathrm{e}}\right) .
$$

Constraints given by (6) to (8) are assumed to be perfect. They derive from a constraints potential

$$
\mathcal{W}_{l}=-\lambda:\left[(1-z) \varepsilon_{\mathrm{A}}+z \varepsilon_{\mathrm{M}}-\varepsilon\right]-\mu\left(\gamma-\sqrt{\frac{2}{3} \varepsilon_{\mathrm{tr}}: \varepsilon_{\mathrm{tr}}}\right)-\nu_{1} z-\nu_{2}(1-z) .
$$

where $\lambda \nu_{1}, \nu_{2}$ and $\mu$ are Lagrange multipliers. $\nu_{1}, \nu_{2}$ and $\mu$, associated with unilateral constraints, must obey the following conditions:

$$
\nu_{1} \geqslant 0, \nu_{1} z=0, \quad \nu_{2} \geqslant 0, \nu_{2}(1-z)=0 \quad \text { and } \mu \geqslant 0, \mu\left(\gamma-\sqrt{\frac{2}{3} \varepsilon_{\mathrm{tr}}: \varepsilon_{\mathrm{tr}}}\right)=0 .
$$

The sum of the free energy $\mathcal{W}$ and of the constraints potential $\mathcal{W}_{l}$ gives the Lagrangian $\mathcal{L}$.

$$
\begin{aligned}
\mathcal{L}= & \mathcal{W}+\mathcal{W}_{l} \stackrel{\text { def }}{=} L\left(\varepsilon, T, \varepsilon_{\mathrm{A}}, \varepsilon_{\mathrm{M}}, z, \varepsilon_{\mathrm{tr}}, \varepsilon_{\mathrm{r}}, \boldsymbol{B}, z_{\mathrm{e}}\right) \\
= & (1-z)\left[\frac{1}{2}\left(\varepsilon_{\mathrm{A}}-\varepsilon_{\mathrm{r}}\right): \boldsymbol{K}_{\mathrm{A}}:\left(\varepsilon_{\mathrm{A}}-\varepsilon_{\mathrm{r}}\right)\right] \\
& +z\left[\frac{1}{2}\left(\varepsilon_{\mathrm{M}}-\varepsilon_{\mathrm{tr}}-\varepsilon_{\mathrm{r}}\right): \boldsymbol{K}_{\mathrm{M}}:\left(\varepsilon_{\mathrm{M}}-\varepsilon_{\mathrm{tr}}-\varepsilon_{\mathrm{r}}\right)+C\left(T, z_{\mathrm{e}}\right)-\frac{2}{3} \boldsymbol{B}: \varepsilon_{\mathrm{tr}}\right]
\end{aligned}
$$




$$
\begin{aligned}
& +G \frac{z^{2}}{2}+\frac{z}{2}[\alpha z+\beta(1-z)]\left(\frac{2}{3} \varepsilon_{\mathrm{tr}}: \varepsilon_{\mathrm{tr}}\right) \\
& -\lambda:\left[(1-z) \varepsilon_{1}+z \varepsilon_{2}-\varepsilon\right]-\mu\left(\gamma-\sqrt{\frac{2}{3} \varepsilon_{\mathrm{tr}}: \varepsilon_{\mathrm{tr}}}\right)-\nu_{1} z-\nu_{2}(1-z),
\end{aligned}
$$

where conditions (10) must be met.

\subsubsection{State equations}

Phase change, martensite orientation, training, as well as the creation of residual strain and internal stress are dissipative processes. Thus, if $\mathcal{A}_{z}, \mathcal{A}_{\mathrm{tr}}, \mathcal{A}_{\mathrm{e}}, \mathcal{A}_{\mathrm{r}}$ and $\mathcal{A}_{\mathrm{B}}$ represent thermodynamic forces associated with state variables $z, \varepsilon_{\mathrm{tr}}, z_{\mathrm{e}}, \boldsymbol{\varepsilon}_{\mathrm{r}}$ and $\boldsymbol{B}$ respectively; only these forces may take non-zero values during a given transformation. Hence the state equations

$$
\begin{aligned}
& \frac{\partial \mathcal{L}}{\partial \varepsilon}=\sigma \quad \Rightarrow \quad \boldsymbol{\lambda}-\boldsymbol{\sigma}=\mathbf{0}, \\
& -\frac{\partial \mathcal{L}}{\partial \varepsilon_{\mathrm{A}}}=\mathbf{0} \quad \Rightarrow \quad(1-z)\left[\boldsymbol{K}_{\mathrm{A}}:\left(\varepsilon_{\mathrm{A}}-\boldsymbol{\varepsilon}_{\mathrm{r}}\right)-\boldsymbol{\lambda}\right]=\mathbf{0}, \\
& -\frac{\partial \mathcal{L}}{\partial \varepsilon_{\mathrm{M}}}=\mathbf{0} \quad \Rightarrow \quad z\left[\boldsymbol{K}_{\mathrm{M}}:\left(\varepsilon_{\mathrm{M}}-\varepsilon_{\mathrm{tr}}-\varepsilon_{\mathrm{r}}\right)-\boldsymbol{\lambda}\right]=\mathbf{0}, \\
& -\frac{\partial \mathcal{L}}{\partial z}=\mathcal{A}_{z} \quad \Rightarrow \quad \mathcal{A}_{z}=\frac{1}{2}\left[\left(\varepsilon_{\mathrm{A}}-\varepsilon_{\mathrm{r}}\right): \boldsymbol{K}_{\mathrm{A}}:\left(\varepsilon_{\mathrm{A}}-\varepsilon_{\mathrm{r}}\right)\right. \\
& \left.-\left(\varepsilon_{\mathrm{M}}-\varepsilon_{\mathrm{tr}}-\varepsilon_{\mathrm{r}}\right): \boldsymbol{K}_{\mathrm{M}}:\left(\varepsilon_{\mathrm{M}}-\varepsilon_{\mathrm{tr}}-\varepsilon_{\mathrm{r}}\right)\right] \\
& -C\left(T, z_{\mathrm{e}}\right)-G z-\left[(\alpha-\beta) z+\frac{\beta}{2}\right]\left(\frac{2}{3} \varepsilon_{\mathrm{tr}}: \varepsilon_{\mathrm{tr}}\right) \\
& -\lambda:\left(\varepsilon_{\mathrm{A}}-\varepsilon_{\mathrm{M}}\right)+\frac{2}{3} \boldsymbol{B}: \varepsilon_{\mathrm{tr}}, \\
& -\frac{\partial \mathcal{L}}{\partial \varepsilon_{\mathrm{tr}}}=\mathcal{A}_{\mathrm{tr}} \quad \Rightarrow \quad \mathcal{A}_{\mathrm{tr}}=z\left\{\boldsymbol{K}_{\mathrm{M}}:\left(\varepsilon_{\mathrm{M}}-\varepsilon_{\mathrm{tr}}-\varepsilon_{\mathrm{r}}\right)-\frac{2}{3}[\alpha z+\beta(1-z)] \varepsilon_{\mathrm{tr}}\right\} \\
& +\frac{2}{3} z \boldsymbol{B}-\frac{2 \mu}{3} \frac{\varepsilon_{\mathrm{tr}}}{\sqrt{\frac{2}{3} \varepsilon_{\mathrm{tr}}: \varepsilon_{\mathrm{tr}}}} \\
& -\frac{\partial \mathcal{L}}{\partial \varepsilon_{\mathrm{r}}}=\mathcal{A}_{\mathrm{r}} \quad \Rightarrow \quad \mathcal{A}_{\mathrm{r}}=(1-z) \boldsymbol{K}_{\mathrm{A}}:\left(\varepsilon_{\mathrm{A}}-\varepsilon_{\mathrm{r}}\right)+z \boldsymbol{K}_{\mathrm{M}}:\left(\varepsilon_{\mathrm{M}}-\varepsilon_{\mathrm{tr}}-\varepsilon_{\mathrm{r}}\right), \\
& -\frac{\partial \mathcal{L}}{\partial \boldsymbol{\lambda}}=\mathbf{0} \quad \Rightarrow \quad(1-z) \varepsilon_{\mathrm{A}}+z \varepsilon_{\mathrm{M}}-\boldsymbol{\varepsilon}=\mathbf{0}, \\
& -\frac{\partial \mathcal{L}}{\partial \boldsymbol{B}}=\mathcal{A}_{\mathrm{B}} \quad \Rightarrow \quad \mathcal{A}_{\mathrm{B}}=\frac{2}{3} z \varepsilon_{\mathrm{tr}} \\
& -\frac{\partial \mathcal{L}}{\partial z_{\mathrm{e}}}=\mathcal{A}_{\mathrm{e}} \quad \Rightarrow \quad \mathcal{A}_{\mathrm{e}}=-z \frac{\partial C\left(T, z_{\mathrm{e}}\right)}{\partial z_{\mathrm{e}}}-\frac{\partial G}{\partial z_{\mathrm{e}}} \frac{z^{2}}{2}
\end{aligned}
$$




$$
-\frac{z}{2}\left[\frac{\partial \alpha}{\partial z_{\mathrm{e}}} z+\frac{\partial \beta}{\partial z_{\mathrm{e}}}(1-z)\right]\left(\frac{2}{3} \varepsilon_{\mathrm{tr}}: \varepsilon_{\mathrm{tr}}\right)-\mu \frac{\partial \gamma}{\partial z_{\mathrm{e}}} .
$$

Equations (12), (13), (14) and (18) allow establishing the following stress-strain relation:

$$
\boldsymbol{\sigma}=\boldsymbol{K}:\left(\varepsilon-z \varepsilon_{\mathrm{tr}}-\varepsilon_{\mathrm{r}}\right) .
$$

$\boldsymbol{K}$ is the equivalent SMA elastic moduli tensor. It is given by

$$
\boldsymbol{K}=\left[(1-z) \boldsymbol{K}_{\mathrm{A}}^{-1}+z \boldsymbol{K}_{\mathrm{M}}^{-1}\right]^{-1} .
$$

\subsubsection{Yield functions and evolution laws}

Residual strain and internal stress depend on the number of loading cycles. Indeed, as shown in figure 2, residual strain is found to increase exponentially with respect to the number of cycles up to an asymptotic value $\varepsilon_{\mathrm{r}}^{\text {sat }}$. From a theoretical point of view, this can be simulated using the following evolution law:

$$
\dot{\varepsilon}_{\mathrm{r}}=\frac{\varepsilon_{\mathrm{r}}^{\mathrm{sat}}}{\tau}\left(\frac{3}{2} \frac{s}{\sigma_{\mathrm{VM}}}\right) \exp \left(-\frac{z_{\mathrm{e}}}{\tau}\right) \dot{z}_{\mathrm{e}}
$$

Residual strain is considered to be deviatoric, not inducing any volume change. $\tau$ is a time constant and $\varepsilon_{\mathrm{r}}^{\text {sat }}$ the maximum residual strain in tension when the hysteresis loop stabilizes, $s$ is the deviatoric part of the stress tensor and $\sigma_{\mathrm{VM}}$ is the equivalent Von Mises measure on the stress tensor.

Dissipation due to the evolution of $\varepsilon_{\mathrm{r}}$ is necessarily positive. Indeed, it can easily be shown that $\mathcal{A}_{\mathrm{r}}$ is unconditionally equal to the stress tensor $\boldsymbol{\sigma} ; \mathcal{A}_{\mathrm{r}}: \dot{\varepsilon}_{\mathrm{r}}$ is hence positive.

Similarly, the evolution of internal stress $\boldsymbol{B}$ is assumed to be governed by the following equation:

$$
\dot{\boldsymbol{B}}=\frac{B^{\mathrm{sat}}}{\tau}\left(\frac{2}{3} \frac{\varepsilon_{\mathrm{tr}}}{\sqrt{\frac{2}{3} \varepsilon_{\mathrm{tr}}: \varepsilon_{\mathrm{tr}}}}\right) \exp \left(-\frac{z_{\mathrm{e}}}{\tau}\right) \dot{z}_{\mathrm{e}}
$$

where $B^{\text {sat }}$ is a positive scalar.

(24) expresses an increase in equivalent internal stress with respect to the number of cycles. Given the expression of the thermodynamic force $\mathcal{A}_{\mathrm{B}}$ associated with state variable $\boldsymbol{B}$, dissipation $\mathcal{A}_{\mathrm{B}}: \dot{\boldsymbol{B}}$ is positive.

Because the evolution of state variables $\boldsymbol{\varepsilon}_{\mathrm{r}}, \boldsymbol{B}$ and $z_{\mathrm{e}}$ is related to that of the martensite volume fraction, one does not need define specific yield functions for each of these variables. Nevertheless, three yield functions: $\mathcal{F}_{z}^{1}, \mathcal{F}_{z}^{2}$ and $\mathcal{F}_{\text {ori }}$ are needed in order to describe forward phase change, reverse phase change and orientation of martensite variants. Thermodynamic forces $\mathcal{A}_{z}$ and $\mathcal{A}_{\text {tr }}$ are chosen to be such that

$$
\begin{gathered}
\mathcal{A}_{z} \in \partial_{\dot{z}} \mathcal{D}, \\
\mathcal{A}_{\mathrm{tr}} \in \partial_{\dot{\varepsilon}_{\mathrm{tr}}} \mathcal{D},
\end{gathered}
$$


where $\mathcal{D}$ is a convex, positive, continuous function that is equal to zero at the origin:

$$
\begin{aligned}
\mathcal{D} & \stackrel{\text { def }}{=} D\left(\dot{z}, \dot{\varepsilon}_{\mathrm{tr}}\right) \\
& =P\left(z, z_{\mathrm{e}}, \dot{z}\right) \dot{z}+R(z) \sqrt{\frac{2}{3} \dot{\varepsilon}_{\mathrm{tr}}: \dot{\varepsilon}_{\mathrm{tr}}},
\end{aligned}
$$

$P\left(z, z_{\mathrm{e}}, \dot{z}\right)$ is given by

$$
P(z, \dot{z})=[a(1-z)+b z] \operatorname{sign} \dot{z}
$$

$a$ and $b$ being $z_{\mathrm{e}}$-dependent parameters:

$$
a=\hat{a}\left(z_{\mathrm{e}}\right) \quad \text { and } \quad b=\hat{b}\left(z_{\mathrm{e}}\right) .
$$

Moreover,

$$
R(z)=z^{2} Y
$$

where $Y$ is a constant material parameter. It is easy to show that $Y, a$ and $b$ are always positive; $\mathcal{D}$ is hence positive. Hence, evolution equations (25) and (26) necessarily satisfy the Clausius-Duheim inequality.

From this point on, austenite and martensite are considered to be homogeneous and isotropic media, having the same Poisson coefficient $\nu$.

$$
\nu_{\mathrm{A}}=\nu_{\mathrm{M}} \stackrel{\text { def }}{=} \nu .
$$

Table 1 summarizes the notations used throughout this paper.

Table 1: Notations used in this paper.

\begin{tabular}{ccr}
\hline Notation & Meaning & Expression \\
\hline \hline$E_{\mathrm{A}}$ & Young modulus of austenite & \\
$E_{\mathrm{M}}$ & Young modulus of martensite & $\left(\frac{1-z}{E_{\mathrm{A}}}+\frac{z}{E_{\mathrm{M}}}\right)^{-1}$ \\
$E_{\mathrm{eq}}$ & Equivalent Young modulus & $\frac{1+\nu}{E_{\mathrm{A}}}$ \\
$\nu$ & Poisson coefficient of the material & $\frac{1+\nu}{E_{\mathrm{M}}}$ \\
$E l_{\mathrm{A}}$ & $\frac{-\nu}{E_{\mathrm{A}}}$ \\
$E l_{\mathrm{M}}$ & $\frac{-\nu}{E_{\mathrm{M}}}$ \\
$P_{\mathrm{A}}$ & & $E l_{\mathrm{M}}-E l_{\mathrm{A}}$ \\
$P_{\mathrm{M}}$ & & $P_{\mathrm{M}}-P_{\mathrm{A}}$ \\
$E l_{\mathrm{MA}}$ & & $\sum_{i} \boldsymbol{M}_{i i}$ \\
$P_{\mathrm{MA}}$ & & \\
$\operatorname{tr} \boldsymbol{M}$ & Trace of symmetrical tensor $\boldsymbol{M}$ & \\
\hline
\end{tabular}




\begin{tabular}{|c|c|c|}
\hline Notation & Meaning & Expression \\
\hline $\operatorname{dev} M$ & Deviator of $\boldsymbol{M}$ & $\boldsymbol{M}-\frac{1}{3}(\operatorname{tr} \boldsymbol{M}) \mathbf{I}$ \\
\hline $\begin{array}{l}M_{\mathrm{VM}} \\
s\end{array}$ & $\begin{array}{c}\text { Von Mises equivalent of } M \\
\text { Stress deviator tensor }\end{array}$ & $\begin{array}{r}\sqrt{\frac{3}{2} \operatorname{dev} \boldsymbol{M}: \operatorname{dev} \boldsymbol{M}} \\
\operatorname{dev} \boldsymbol{\sigma}\end{array}$ \\
\hline$\sigma_{\mathrm{VM}}$ & Von Mises equivalent stress & $\sqrt{\frac{3}{2} s: s}$ \\
\hline$\mu_{\mathrm{A}}$ & Austenite shear modulus & $\frac{E_{\mathrm{A}}}{2(1+\nu)}$ \\
\hline$\mu_{\mathrm{M}}$ & Martensite shear modulus & $\frac{E_{\mathrm{M}}}{2(1+\nu)}$ \\
\hline$\mu_{\mathrm{eq}}$ & Equivalent shear modulus & $\frac{L_{\mathrm{eq}}}{2(1+\nu)}$ \\
\hline
\end{tabular}

Using equations (25) and (26), the following expressions of $\mathcal{F}_{z}^{1}, \mathcal{F}_{z}^{2}$ and $\mathcal{F}_{\text {ori }}$ can be established:

$$
\begin{aligned}
\mathcal{F}_{z}^{1}=\{ & \left.\frac{1}{3} E l_{\mathrm{MA}} \sigma_{\mathrm{VM}}^{2}+\frac{1}{2}\left(\frac{1}{3} E l_{\mathrm{MA}}+P_{\mathrm{MA}}\right)(\operatorname{tr} \boldsymbol{\sigma})^{2}-C\left(T, z_{\mathrm{e}}\right)\right\} \\
& +\left(\boldsymbol{\sigma}+\frac{2}{3} \boldsymbol{B}\right): \varepsilon_{\mathrm{tr}} \\
& -(G+b) z-a(1-z)-\left[(\alpha-\beta) z+\frac{\beta}{2}\right]\left(\frac{2}{3} \varepsilon_{\mathrm{tr}}: \varepsilon_{\mathrm{tr}}\right), \\
\mathcal{F}_{z}^{2}=- & \left\{\frac{1}{3} E l_{\mathrm{MA}} \sigma_{\mathrm{VM}}^{2}+\frac{1}{2}\left(\frac{1}{3} E l_{\mathrm{MA}}+P_{\mathrm{MA}}\right)(\operatorname{tr} \boldsymbol{\sigma})^{2}-C\left(T, z_{\mathrm{e}}\right)\right\} \\
& -\left(\boldsymbol{\sigma}+\frac{2}{3} \boldsymbol{B}\right): \varepsilon_{\mathrm{tr}} \\
& +(G-b) z-a(1-z)+\left[(\alpha-\beta) z+\frac{\beta}{2}\right]\left(\frac{2}{3} \varepsilon_{\mathrm{tr}}: \varepsilon_{\mathrm{tr}}\right), \\
\mathcal{F}_{\text {ori }}=\|(\boldsymbol{\sigma} & \left.+\frac{2}{3} \boldsymbol{B}\right)-\frac{2}{3}[\alpha z+\beta(1-z)] \varepsilon_{\mathrm{tr}}-\frac{2 \mu}{3 z} \frac{\varepsilon_{\mathrm{tr}}}{\sqrt{\frac{2}{3} \varepsilon_{\mathrm{tr}}: \varepsilon_{\mathrm{tr}}}} \|_{\mathrm{VM}}-z Y .
\end{aligned}
$$

It is worth noting that in each of these expressions, a quantity $\frac{2}{3} \boldsymbol{B}$, proportional to internal stress due to training, is added to the stress tensor $\boldsymbol{\sigma}$. Thus, both $\boldsymbol{B}$ and $\boldsymbol{\sigma}$ have similar effects as to phase change and martensite orientation.

Phase change evolution laws obey certain conditions: 
- If $\mathcal{F}_{z}^{1}<0$ and $\mathcal{F}_{z}^{2}<0$, no phase change can occur. Hence,

$$
\dot{z}=0 ;
$$

- If forward phase change is triggered, $\mathcal{F}_{z}^{1}$ is equal to zero. In this case, $\dot{z}$ is equal to zero if $\dot{\mathcal{F}}_{z}^{1}<0$; otherwise, $\dot{z}$ is given by the consistency condition $\dot{\mathcal{F}}_{z}^{1}=0$;

- If reverse phase change is triggered, $\mathcal{F}_{z}^{2}$ is equal to zero. In this case, $\dot{z}$ is equal to zero if $\dot{\mathcal{F}}_{z}^{2}<0$; otherwise, $\dot{z}$ is given by the consistency condition $\dot{\mathcal{F}}_{z}^{2}=0$. Let

$$
\boldsymbol{X} \stackrel{\text { def }}{=}\left(\boldsymbol{s}+\frac{2}{3} \boldsymbol{B}\right)-\frac{2}{3}[\alpha z+\beta(1-z)] \varepsilon_{\mathrm{tr}}-\frac{2 \mu}{3 z} \frac{\varepsilon_{\mathrm{tr}}}{\sqrt{\frac{2}{3} \varepsilon_{\mathrm{tr}}: \varepsilon_{\mathrm{tr}}}} .
$$

The yield function $\mathcal{F}_{\text {ori }}$, associated with martensite orientation, can be written as

$$
\mathcal{F}_{\text {ori }}=X_{\mathrm{VM}}-z Y \text {. }
$$

Evolution of local inelastic strain tensor $\varepsilon_{\operatorname{tr}}$ satisfies the normality law:

$$
\begin{aligned}
\dot{\varepsilon}_{\mathrm{tr}} & =\eta \frac{\partial \mathcal{F}_{\mathrm{ori}}}{\partial \boldsymbol{X}} \\
& =\frac{3}{2} \eta \frac{\boldsymbol{X}}{X_{\mathrm{VM}}} .
\end{aligned}
$$

In the above equation, $\eta$ is a positive scalar satisfying Kuhn-Tucker conditions:

$$
\eta \geqslant 0, \quad \mathcal{F}_{\text {ori }} \leqslant 0 \text { and } \quad \eta \mathcal{F}_{\text {ori }}=0 .
$$

Let $\sigma_{\mathrm{rs}}$ and $\sigma_{\mathrm{rf}}$ be orientation start and finish stresses of self-accommodating martensite:

- When orientation starts, yield function $\mathcal{F}_{\text {ori }}$ is necessarily equal to zero for $\varepsilon_{\text {tr }}=\mathbf{0}$, $\|\boldsymbol{\sigma}+\boldsymbol{B}\|_{\mathrm{VM}}=\sigma_{\mathrm{rs}}$ and $\mu=0$. It follows that

$$
Y=\sigma_{\mathrm{rs}}
$$

- When orientation is complete, $\mathcal{F}_{\text {ori }}=0$ for $\sqrt{\frac{2}{3} \varepsilon_{\mathrm{tr}}: \varepsilon_{\mathrm{tr}}}=\gamma$. If $\sqrt{\frac{2}{3} \varepsilon_{\mathrm{tr}}: \varepsilon_{\mathrm{tr}}}$ tends towards $\gamma$ with lower values, $\mu$ is equal to zero for $\|\boldsymbol{\sigma}+\boldsymbol{B}\|_{\mathrm{VM}}=\sigma_{\mathrm{rf}}$. In case of uniaxial tension, it follows that

$$
\left|\sigma_{\mathrm{rf}}-\alpha \gamma\right|=Y
$$

the above equation, together with equation (40), gives $\alpha$ as a function of $\gamma$ :

$$
\alpha=\frac{\sigma_{\mathrm{rf}}-\sigma_{\mathrm{rs}}}{\gamma} .
$$

- When austenite transforms into oriented martensite, orientation is complete when stress becomes greater or equal to $\sigma_{\mathrm{rf}}$. Particularly, if stress tends towards $\sigma_{\mathrm{rf}}$ with lower values, $\mu$ remains equal to zero. In this case,

$$
z=0, \quad\|\boldsymbol{\sigma}+\boldsymbol{B}\|_{\mathrm{VM}}=\sigma_{\mathrm{rf}}, \quad \sqrt{\frac{2}{3} \varepsilon_{\mathrm{tr}}: \varepsilon_{\mathrm{tr}}}=\gamma \quad \text { and } \quad \mu=0 .
$$

In case of uniaxial tension,

$$
\beta=\frac{\sigma_{\mathrm{rf}}}{\gamma}
$$


$\alpha$ and $\beta$ are both functions of $z_{\mathrm{e}}$ due to their dependence on $\gamma$.

Finally, explicit expressions of the evolution laws can be derived using the consistency conditions.

\subsection{Numerical simulation}

Figure 4 shows the experimental response of a NiTi test sample to repeated uniaxial tension.
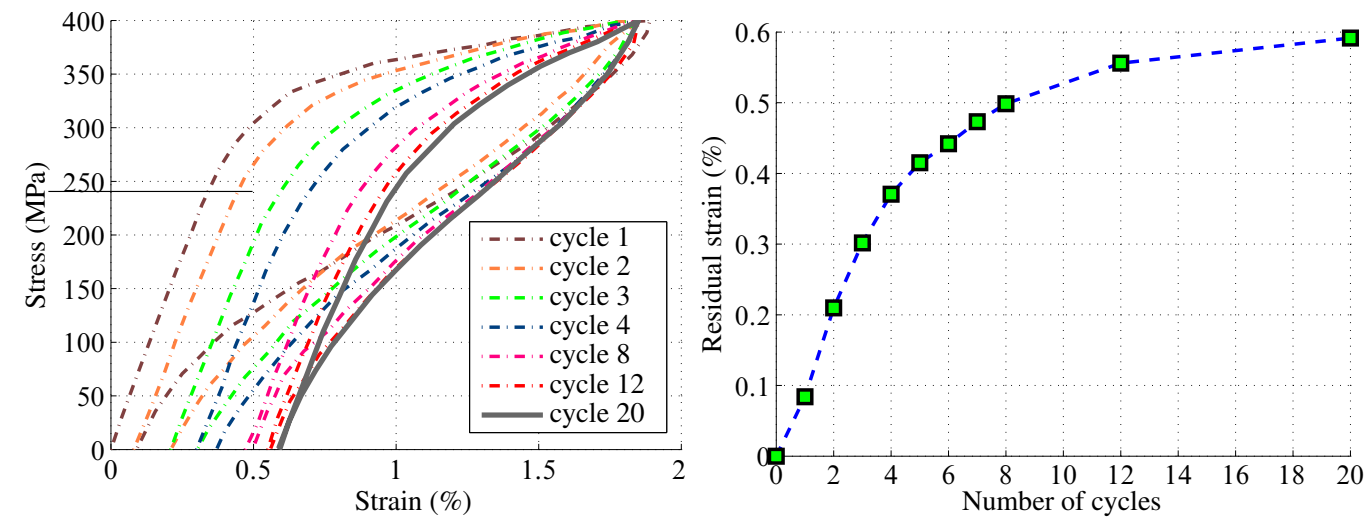

Fig. 4. Experimental stress-strain response of a NiTi wire to repeated tension. The stabilized

Fig. 5. Residual strain evolution with respect cycle is shown in continuous line.

Residual strain evolution with respect to the number of cycles is shown in figure 5 . The Nickel-Titanium used in the experiments has an orientation start stress of $80 \mathrm{MPa}$ and

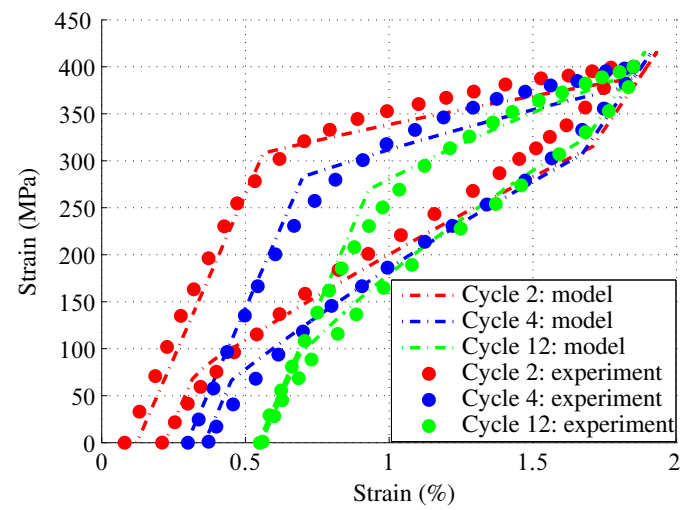

Fig. 6. Experimental vs numerical results. Evolution of the superelastic loop with the number of cycles. 
an orientation finish stress equal to $160 \mathrm{MPa}$. Reverse transformation finish temperature of the untrained material at zero stress is equal to $42^{\circ}$; this temperature does not evolve considerably with the number of cycles.

Figure 6 shows good agreement between experimental and numerical results in the case of repeated tension. For clarity, only loops 2, 4 and 12 are shown.

Figure $7(\mathrm{a})$ illustrates the evolution of internal strain $B$ with respect to the cumulated martensite volume fraction $z_{\mathrm{e}}$. Stress-strain response for all 20 loading cycles is shown in figure $7(\mathrm{~b})$.

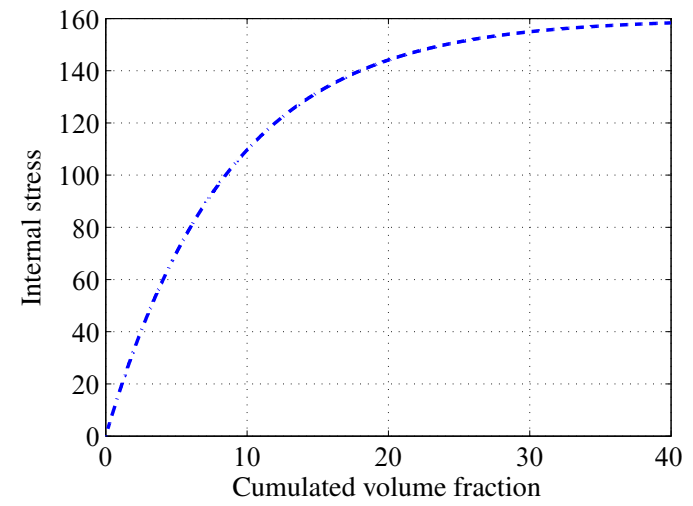

(a) Evolution of internal stress.

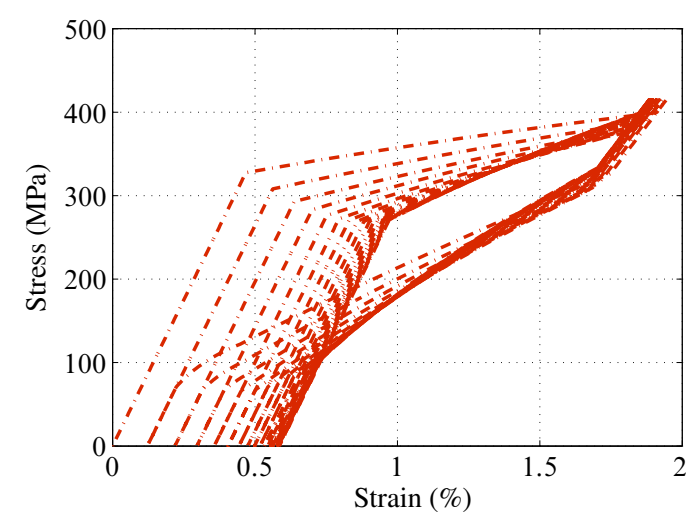

(b) Stress-strain response cycles.

Fig. 7. Prediction of Nickel-Titanium response to repeated tension.

The next section is devoted to the presentation of an energy approach of the fatigue of shape memory alloys. It is shown how the cyclic model presented in the previous section can be combined with the fatigue criterion in order to perform numerical calculations of the fatigue parameters necessary for the evaluation of life time of structures made on SMAs.

\section{AN ENERGY APPROACH OF THE FATIGUE OF SHAPE MEMORY ALLOYS}

Fatigue of shape memory alloys is generally explained by the creation and propagation of defects within the material at the microscopic level [11]. A more rigorous understanding is complicated, however, due to phenomena like formation of residual martensite [25] and local phase change at the tip of microscopic cracks [30], resulting in slower crack propagation in martensite [4].

Because of similar damage creation and propagation mechanisms, it is interesting to investigate the fatigue of SMAs within a framework similar to that of usual elastoplastic materials (like steel). It is, hence, useful to distinguish between:

- Low-cycle fatigue;

- Finite fatigue life in high cycle fatigue;

- High cycle fatigue (infinite life). 
This section focuses on low-cycle fatigue associated with cyclic superelasticity.

\subsection{Experimental analysis}

\subsubsection{Material and thermomechanical treatment}

The material is a $51,3 \% \mathrm{Ti}-48,7 \% \mathrm{Ni}$ in mass Nickel-Titanium with a grain size between $60 \mu \mathrm{m}$ and $70 \mu \mathrm{m} c f$. figure 8 . Phase change temperatures at zero stress are as follows: $M_{\mathrm{f}}^{0}=25^{\circ} \mathrm{C}, M_{\mathrm{s}}^{0}=39^{\circ} \mathrm{C}, A_{\mathrm{s}}^{0}=29^{\circ} \mathrm{C}$ and $A_{\mathrm{f}}^{0}=42{ }^{\circ} \mathrm{C}$.

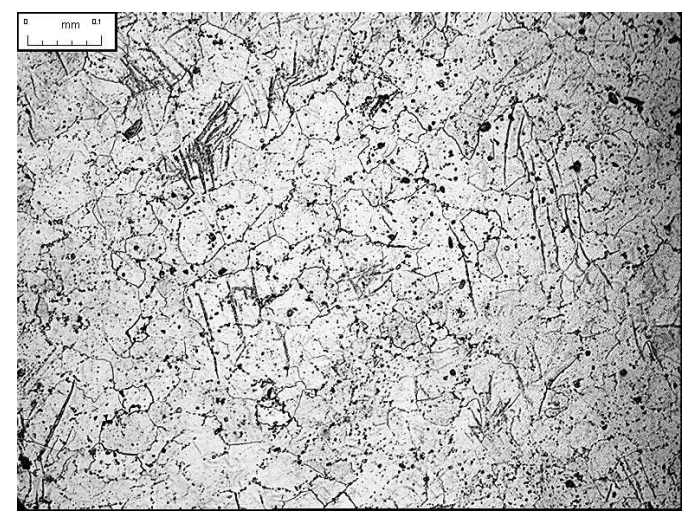

Fig. 8. Metallographic structure of the NiTi alloy.

All the test specimens were cold worked up to $20 \%$ in tension, then heat treated at $400{ }^{\circ} \mathrm{C}$ for one hour. This kind of treatment increases the plastic yield limit of the material while improving its superelasticity [31].

\subsubsection{Experimental setup}

The testing machine used is a force controlled MTS810/100KN. The strains are measured using an MTS extensometer model 632-13C-21. Load and extensometer signals are captured by an MTS TestStarII data acquisition board and processed by a computer. Experiments are Stress-controlled push-pull tests with a constant amplitude $\sigma_{\mathrm{a}}$. They are carried out at a frequency of $0.3 \mathrm{~Hz}$. In order to ensure that the tests are performed in the pseudoelatic domain the temperature is kept constant at $T=50{ }^{\circ} \mathrm{C}$ which is higher than the austenite finish temperature $A_{\mathrm{f}}^{0}$. The experiments were conducted in a SERVATHIN hermetic enclosure where the temperature can be regulated and kept constant through a range values from $-50{ }^{\circ} \mathrm{C}$ to $200{ }^{\circ} \mathrm{C}$. Maximum stress was kept below the critical stress for slip (750 MPa) to ensure that no macroscopic plastic deformations occurs. The tests were take through to rupture of the specimen.

In order to examine the effect of mean stress on the fatigue of Nickel-Titanium,

three load ratios $\left(R=\frac{\sigma_{\min }}{\sigma_{\max }}\right)$ were considered, equal to $0,0.2$ and -1 repesctively. The geometries of the specimens we used are illustrated in figures 9(a) and 9(b). 


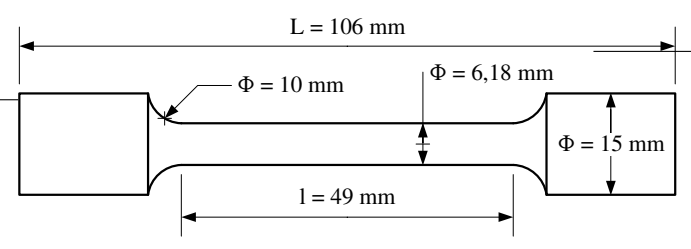

(a) For $R=0$.

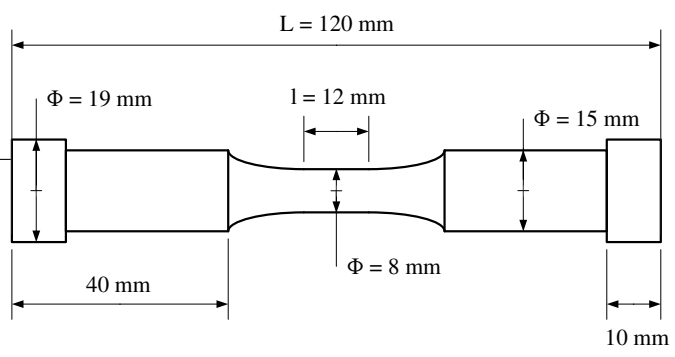

(b) For $R=0.2,-1$.

Fig. 9. Geometries of specimens used for cyclic experiments.

\subsection{Results and discussions}

\subsection{1. $S$ - $N$ curves}

The S-N curves (Wöhler curves) relating the number of cycles to failure under uniaxial loading to the amplitude of the applied stress obtained for these experiments are shown on figure 10 .

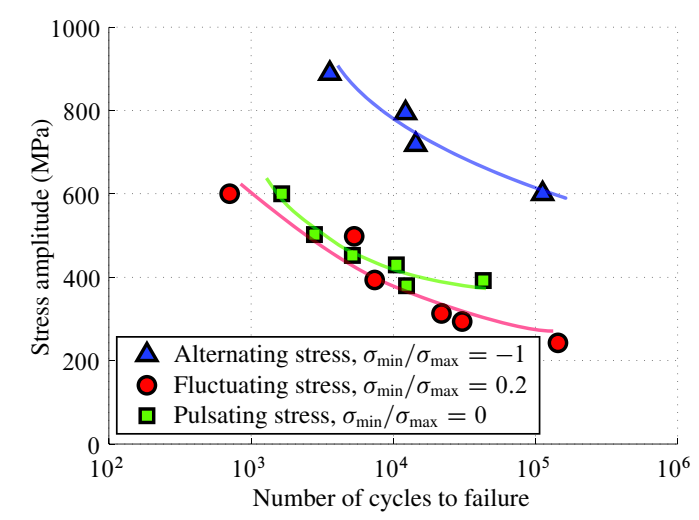

Fig. 10. Wöhler curves of Nickel-Titanium for different mean stress values.

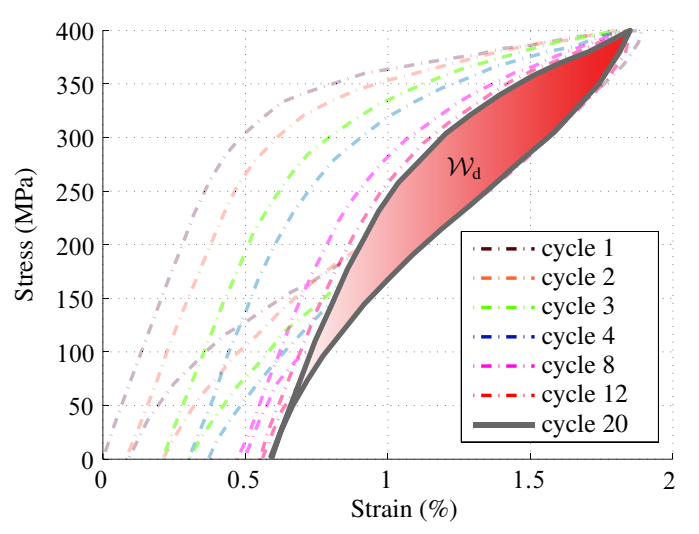

Fig. 11. Stabilization of the amount of dissipated energy per cycle, $\mathcal{W}_{\mathrm{d}}$, with the number of cycles: experimental result.

The effect of mean stress on fatigue life can be observed. Indeed, a higher mean stress corresponds to a lower number of cycles to failure.

These Wöhler curves can be used for fatigue life prediction when the applied loading is uniaxial and for a given mean stress. They are inadequate, however, for fatigue analysis of shape memory structures under multiaxial loading. 


\subsubsection{Low-cycle fatigue life prediction for superelastic SMAs}

Existing SMA low-cycle fatigue life prediction models are mostly of the MansonCoffin type: the number of cycles to failure is related to the amplitude of plastic strain.

As early as 1979, showed that, for different types of shape memory alloys, fatigue life of wires follows the Manson-Coffin law. This result has been confirmed in several subsequent papers $[27,30]$.

Even though multiaxial loading can be accounted for theoretically by means of a generalized Manson-Coffin relationship using equivalent plastic strain, the ability to predict 3D structure failure using this approach has not been proved.

In the scope of this paper, an energy-based approach is used for estimating low-cycle fatigue life of superelastic SMAs.

This is inspired from [3], where a similar approach was successfully applied on cast iron. Indeed, superelastic hysteresis stabilization for a shape memory material is similar to plastic shakedown of cast iron. In both cases, inelastic (plastic deformation for iron cast and transformation strain for SMAs) deformation is confined but the material continues to dissipate energy. Energy dissipation is usually explained, in the case of superelastic SMAs, by strain incompatibilities across the boundaries of the grains [12], which in time lead to the creation and accumulation of deffects at the boundaries [11].

In this paper, the martensitic transformation, responsible for the creation of martensite grains, is accounted for using state variables $z$ and $\varepsilon_{\mathrm{tr}}$. Given the above interpretation of energy dissipation in SMAs, the inelastic deformation $z \varepsilon_{\mathrm{tr}}$, being proportional to the number of martensite grain boundaries (through state variable $z$ ) and to the level of orientation of martensite variants within these grains, seems an adequate parameter for predicting fatigue failure of superelastic SMAs.

The amount of dissipated energy per loading cycle, $\mathcal{W}_{\mathrm{d}}$, is given by

$$
\mathcal{W}_{\mathrm{d}}=\oint \sigma: d \varepsilon
$$

Once the material response stabilizes, this energy becomes constant, as shown by the experimental result given in figure 11 (stress ratio $=0$ ).

In the case of the studied Nickel-Titanium, an example of the evolution of the dissipated energy with respect to the number of cycles is illustrated in figure 12 . Even though the use of dissipated energy per cycle for fatigue life prediction has been criticized $[5,20]$, its usefulness in practice is well proven: the work of [3] has been successfully applied to predicting failure of automotive components subjected to complex thermomechanical loading.

Figure 13 represents the amount of dissipated energy per cycle, $\mathcal{W}_{\mathrm{d}}$, with respect to the number of cycles to failure $N_{\mathrm{f}}$, using a $\log -\log$ scale.

The figure shows a quasi-linear dependence of $\log \mathcal{W}_{\mathrm{d}}$ on $\log N_{\mathrm{f}}$ for several values of the mean stress. It is, hence, interesting to approximate experimental results using the following curve [17]:

$$
\mathcal{W}_{\mathrm{d}}=\alpha N_{\mathrm{f}}^{\beta}
$$

where $\alpha$ and $\beta$ are material parameters. Numerical results are in good agreement with experimental data for $\alpha=11$ and $\beta=-0.377$, as shown in figure 14 . 


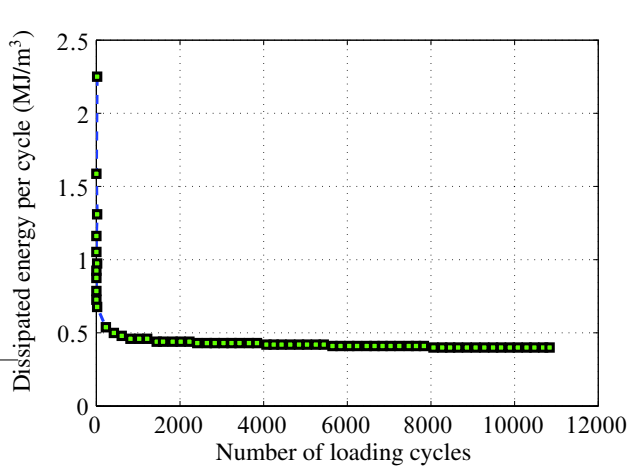

Fig. 12. Dissipated energy per cycle vs number of loading cycles: experimental result for $\Delta \sigma=400$ Mpa.

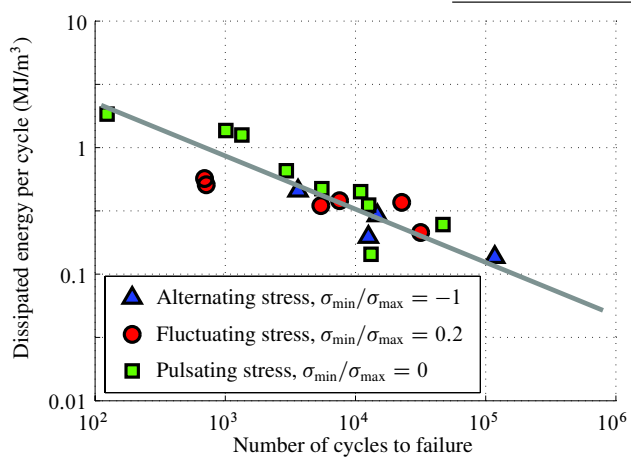

Fig. 14. Numerical vs experimental results representing dissipated energy per cycle as a function of the number of cycles to failure in a log-log scale.

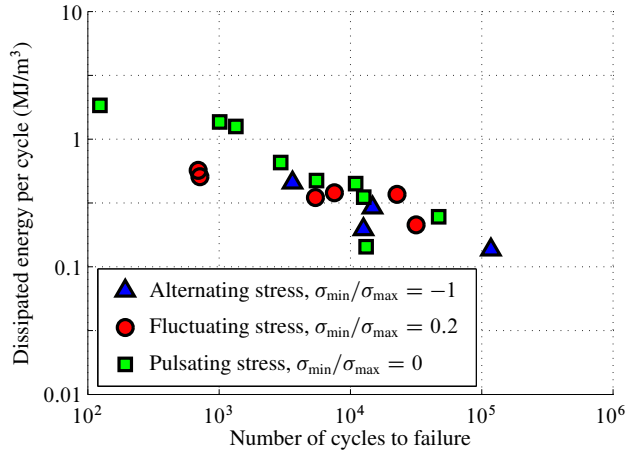

Fig. 13. Dissipated energy per cycle vs number of cycles to failure.

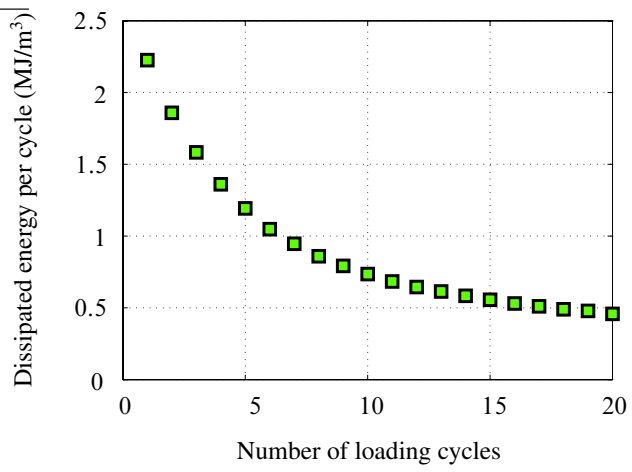

Fig. 15. Numerical results. Dissipated energy per cycle with respect to the number of cycles: numerical result for $\Delta \sigma=400 M p a$.

Expression (46) may readily be used for 3D structure analysis because dissipated energy per cycle is well defined and its calculation is straightforward.

The stabilized cycle and the corresponding amount dissipated energy to the stabilized cycle can be numerically determined using the model presented in this paper as shown in figure 15.

The number of cycles to failure of a SMA structure can be estimated using the suggested fatigue criterion. It is important to note, however, that the validity of this criterion has only been proven in the case of uniaxial loading; its ability to predict failure of structures subjected to complicated loading conditions remains to be established. 


\section{CONCLUSION}

In the first part of this paper a model capable of simulating several phenomena associated with shape memory materials subjected to cyclic loading is presented. The modeling process is based on a simple observation: on the macroscopic level, SMA training can be interpreted as a thermomechanically-induced transition from an unstable, virgin material configuration into a stable one. From a theoretical point of view, it is easy to account for this transition by making some of the model parameters depend on a cumulated martensite volume fraction which evolves with the applied loading.

Inelastic residual strain, which appear during repeated phase change, is accounted for by introducing a state variable similar to plastic deformation strain of classical elastoplastic materials. Numerical results show good agreement with available experimental data.

The second part of the paper investigates the fatigue of SMAs by analogy with plastic fatigue. It has been shown that the dissipated energy at the stabilized cycle during a cyclic loading is a relevant parameter for fatigue life prediction. A relationship between this parameter and the number of cycle to failure has been derived from experimental results. It has also been shown that the cyclic model can be combined with the fatigue criterion in order to predict low-cycle failure of superelastic shape memory structures.

Nevertheless, it is clear that the model must be improved on some points. First, it is important to investigate the fatigue of SMAs criterion for another type of loading, namely torsion. Second, it is interesting to check the validity of the model against experimental results for complex structures under complex loading. This work is undertaken and will be presented in future papers.

\section{REFERENCES}

[1] R. Abeyaratne and S.J Kim, Cyclic effects in shape-memory alloys: a one-dimensional continuum model. International Journal of Solids and Structures, 34:3273-3289, 1997.

[2] F. Auricchio, S Marfia, and E. Sacco. Modelling of SMA materials: training and two way memory effect. Computers and Structures, 81:2301-2317, 2003.

[3] E. Charkaluk, A. Bignonnet, A Constantinescu, and K Dang Van. Fatigue design criterion for structures under thermomechanical loading. Fatigue and Fracture of Engineering Materials and Structures, 25:1199-206, 2002.

[4] G. Eggeler, E. Hornbogen, A. Yawny, A. Heckmann, and M. Wagner. Structural and functional fatigue of NiTi shape memory alloys. Materials Science and Engineering A, 378:24-33, 2004.

[5] G. R. Halford. The energy required for fatigue. Journal of Materials, 1:3-18, 1966.

[6] S. Leclerq and C. Lexcellent. A general macroscopic description of the thermomechanical behavior of shape memory alloys. Journal of the Mechanics and Physics of Solids, 44(6): 953-980, 1996.

[7] C. Lexcellent and G. Bourbon. Thermodynamical model of cyclic behaviour of $\mathrm{Ti}-\mathrm{Ni}$ and $\mathrm{Cu}-\mathrm{Zn}-\mathrm{Al}$ shape memory alloys under isothermal undulated tensile tests. Mechanics of Materials, 24:59-73, 1996.

[8] T.J. Lim and D.L. McDowell. Cyclic thermomechanical behavior of a polycrystalline pseudoelastic shape memory alloy. Journal of the Mechanics and Physics of Solids, 50:651-676, 2002 . 
[9] Y. Liu, Z. Xie, and J. Van Humbeeck. Cyclic deformation of NiTi shape memory alloys. Materials Science and Engineering A, 273-275:673-678, 1999.

[10] A. L. McKelvey and R.0. Ritchie. Fatigue-crack propagation in nitinol, a shape-memory and superelastic endovascular stent material. Journal of Biomedical Materials Research, 47: 301-308, 1999.

[11] K. N. Melton and O. Mercier. The effect of the martensitic phase transformation on the low cycle fatigue behaviour of polycrystalline $\mathrm{Ni}-\mathrm{Ti}$ and $\mathrm{Cu}-\mathrm{Zn}-\mathrm{Al}$ alloys. Materials Science and Engineering, 40:81-87, 1979.

[12] K. N. Melton and O. Mercier. Fatigue life of CuZnAl alloys. Scripta Metallurgica, 13:73-75, 1979.

[13] K. N. Melton and O. Mercier. Fatigue of NiTi thermoelastic martensites. Acta Metallurgica, $27: 137-144,1979$.

[14] T. IGO Y. Miyazaki, S. Imai and K. Otsuka. Effect of cyclic deformation on the pseudoelasticity characteristics of ti-ni alloys. Metall. Trans., 17a:15-20, 1986.

[15] N.B. Morgan. Medical shape memory alloy applications - the market and its products. Materials Science and Engineering A, 378:16-23, 2004.

[16] Z. Moumni. Sur la modélisation du changement de phase à l'état solide. PhD thesis, École Nationale Supérieure des Ponts et Chaussées, 1995.

[17] Z. Moumni, A. Van Herpen, and P. Riberty. Fatigue analysis of shape memory alloys: energy approach. Smart Materials and Structures, 14:S287-S292, 2005.

[18] Z. Moumni, W. Zaki, and Q. S. Nguyen. Theoretical and numerical modeling of solid-solid phase change: Application to the description of the thermomechanical behavior of shape memory alloys. International Journal of Plasticity, 2008.

[19] E. Patoor and M. Berveiller. Lois de comportement et calcul de structures en alliage à mémoire de forme. In Technologie des Alliages à Mémoire de Forme, chapter 9, pages 195224. HERMES, 1993.

[20] A. Pineau and P Pộtrequin. La fatigue des matộriaux et des structures, $2^{e}$ ộdition revue et augmentọe, chapter 4, pages 155-214. Hermes, Paris, 1997.

[21] G.A. Porter, P.K. Liaw, T.N. Tiegs, and K.H. Wu. Fatigue and fracture behavior of NickelTitanium shape-memory alloy reinforced aluminum composites. Materials Science and Engineering A, 314:186-193, 2001.

[22] W. Predki, M. Klönne, and A. Knopik. Cyclic torsional loading of pseudoelastic NiTi shape memory alloys: damping and fatigue failure. Materials Science and Engineering A, 417: $182-189,2006$.

[23] B. Raniecki, C. Lexcellent, and K. Tanaka. Thermodynamic models of pseudoelastic behaviour of shape memory alloys. Archives of Mechanics, 44:261-284, 1992.

[24] J. A. Shaw and S. Kyriakides. Thermomechanical aspects of NiTi. Journal of the Mechanics and Physics of Solids, 43(8):1243-1281, 1995.

[25] N. Siredey, A. Hautcoeur, and A. Eberhardt. Lifetime of superelastic $\mathrm{Cu}-\mathrm{Al}-\mathrm{Be}$ single crystal wires under bending fatigue. Materials Science and Engineering A, 396:296-301, 2005.

[26] K. Tanaka, F. Nishimura, T. Hayashi, H. Tobushi, and C. Lexcellent. Phenomenological analysis on subloops and cyclic behavior in shape memory alloys under mechanical and/or thermal loads. Mechanics of Materials, 19:281-292, 1995.

[27] Davidson S. Tolomeo, D. and M. Santinoranont. Cyclic properties of superelastic Nitinol: design implications. In Shape memory and superelastic technologies conference proceedings, 2001. 
[28] R. Vaidynanathan, D.C. Dunand, and U. Ramamurty. Fatigue crack-growth in shape-memory Ni-Ti and NiTi-TiC composites. Materials Science and Engineering A, 289:208-216, 2000.

[29] J. Van Humbeeck. cycling effects, fatigue and degradation of shape memory alloys. Journal de physique, colloque C4, 4:189-197, 1991.

[30] M. Wagner, T. Sawaguchi, G. Kausträter, D. Höffken, and G. Eggeler. Structural fatigue of pseudoelastic NiTi shape memory wires. Materials Science and Engineering A, 378:105-109, 2004.

[31] C.M. Wayman and K. Otsuka, editors. Shape Memory Alloys. Cambridge University Press, 1999.

[32] W. Zaki. Comportement thermo-mécanique des matériaux à mémoire de forme. $\mathrm{PhD}$ thesis, École Polytechnique, 2006.

[33] W. Zaki and Z. Moumni. A three-dimensional model of the thermomechanical behavior of shape memory alloys. Journal of the Mechanics and Physics of Solids, 55:2455-2490, 2007.

[34] W. Zaki and Z. Moumni. A 3d model of the cyclic thermomechanical behavior of shape memory alloys. Journal of the Mechanics and Physics of Solids, 55:2427-2454, 2007.

[35] Z.Bo and D.Lagoudas. Thermomechanical modeling of polycrystalline SMAs under cyclic loading, part I: theoretical derivations. International Journal of Engineering Science $\mathbf{3 7}$ (1999) 1089-1140, 1999.

Received September 30, 2009

\section{ỨNG XỬ TUẦN HOÀN VÀ PHƯƠNG PHÁP NĂNG LƯợNG VỀ MỎI CỦA CÁC HợP KIM NHỚ HÌNH DẠNG}

Bài báo này trình bày một tiêu chuẩn mà có thể được sử dụng trong việc phân tích và thiết kế các kết cấu tạo bởi các hợp kim nhớ hình dạng (SMAs) chịu tải trọng tuần hoàn. Một cách thực nghiệm thì một phản ứng tương tự như shakedown dẻo sẽ được quan sát: trong các chu kỳ đầu tiên thì đường cong ứng suất-biến dạng sẽ mô tả vòng lặp trễ mà nó phát triển trong vài chu kỳ đầu tiên trước khi ổn định. Bằng việc ứng dụng một sự tương tự trong mỏi dẻo thì năng lượng tiêu tán của một chu kỳ là một tham số thích hợp cho việc ước lượng số chu kỳ để làm hỏng các vật liệu như vậy. Tuân theo những quan sát như vậy chúng tôi trình bày một ứng dụng của mô hình tuần hoàn mà đó được tác giả phát triển trước đó trong khuôn khổ các vật liệu tiêu chuẩn tổng quát hóa với các ràng buộc Moumni (1995) để đánh giá tham số này. Các mô phỏng số đó được trình bày cùng với các số liệu kiểm chứng bằng thực nghiệm trong trường hợp siêu dẻo tuần hoàn. Từ khóa: tải trọng tuần hoàn, biến dạng dư, ứng suất nội, tiêu tán, mỏi 